\title{
Zur geplanten Fortsetzung des Textes
}

\section{Ein Nachwort}

Pierre Bühler

In einer Mappe zum zweiten Teil des Manuskripts finden sich verschiedene Unterlagen, die Gerhard Ebeling angesammelt hat, um seine Arbeit fortzuführen:

- verschiedene Notizenbündel (etwa zum Lebensbegriff als Schlüssel der Theologie, anscheinend in Verbindung mit einem Vortrag im Löwensteiner Kreis; zur Beziehung mit Ernst Fuchs, vor allem in Anlehnung an Fuchs' Buch Das Wagnis des Glaubens (Neukirchen-Vluyn 1979);

- zur Thematik von Wort und Glaube als Konstante seines Werks;

- frühe Skizzen des Gesamtaufbaus;

- erste kurze und undatierte Versuche zum zweiten Teil, teils handschriftlich, teils bereits in Maschinenschrift.

Aufgrund dieses Materials lässt sich nicht nur erschliessen, wie Ebeling die weitere Arbeit geplant, sondern auch, mit welchen Schwierigkeiten er dabei zu kämpfen hatte.

Bereits in den frühen Skizzen zeigt sich die Zweiteilung in einen eher biographischen und einen stärker theologischen Teil. Früh schon heissen die betreffenden Überschriften "Wegstrecken" und "(theologische) Brennpunkte ".Werden die biographischen Wegstrecken in zwei Phasen aufgeteilt - -Theologiestudium und Dienst in der Bekennenden Kirche" und "Stadien der akademischen Lehrtätigkeit" - so geht es bei den theologischen Brennpunkten immer um vier Aspekte, die zwar eindeutig feststehen, jedoch recht unterschiedlich umschrieben werden.

Zum Begriff der "Brennpunkte" heisst es in einer ersten, zunächst ganz kurzen Fassung der Einleitung in den zweiten Teil:

" $\mathrm{Zu}$ den Brennpunkten, auf die sich meine theologische Arbeit konzentriert, rechne ich diejenigen gezielten Vorhaben, welche doch vielerlei Einzelthemen übergreifen und dabei auch einander überschneiden können. Kenntlich sind sie an den Titeln mehrbändiger Werke, ob diese nun viele Aufsätze in sich vereinen oder ein grosses Thema in Teilbände zerle- 
gen oder von kleineren entsprechenden Publikationen begleitet sind. [...] So kommt es zu vier Brennpunkten, die mehr oder weniger das ganze Opus durchziehen: Wort und Glaube, Fundamentaltheologie, Dogmatik des christlichen Glaubens und Lutherstudien.»

In den ersten Skizzen gibt es bei einzelnen Brennpunkten gewisse Schwankungen: Neben "Wort und Glaube» stehen öfters "Gal" (die Galaterbrief-Vorlesung) und "Pred" (die Predigtbände), einmal auch "Wort und Gott" und »Theologische Sprachlehre». Bei "Fundamentaltheologie« wird einmal mit "Enzyklop." ergänzt. Auch beim vierten Brennpunkt schwanken die Bezeichnungen: Neben "Lutherstudien" kann auch stehen "Luther", "(Fragmente zur) Theol. Luthers" oder "Orientierung an Luther".

An diesen Varianten zeigt sich bereits, dass der Begriff "Brennpunkt" nicht so eindeutig ist, wie man meinen könnte. Auf jedem Fall gibt es auf einem Blatt mit der Überschrift »Erneute Überlegungen zum 2. (systematischen) Teil« folgende Notizen:

"An ein Ziel kommen? So dass die theol. Aufgabe abgeschlossen ist/wäre?

Die hist. Theol. bleibt auf jeden Fall ein Bruchstück.

Die syst. Theol. wohl erst recht?

Man könnte meinen, die Addition der 4 Themen

Wort und Glaube

Fund.theol.

Dogm.

Luther

ergäbe ein Ganzes.

Doch handelt es sich bei den 4 Richtungsangaben

teils (nur) um Überschneidungen

teils um Bruchstücke."

In Verbindung mit der ersten Kurzfassung der Einleitung hat Ebeling bereits den ersten Brennpunkt, "Wort und Glaube«, in Angriff genommen. Dazu gibt es in den Notizen auch am meisten Material, während $\mathrm{zu}$ den drei anderen Brennpunkten nichts zu finden ist.

So heisst es denn im ersten Versuch zum Brennpunkt "Wort und Glaube«:

"Das Erscheinen des noch unnummerierten ersten Aufsatzbandes 'Wort und Glauber im Jahre 1960 war in mehrfacher Hinsicht in meiner Biographie verankert. Das 
Vorwort läßt dies deutlich erkennen. ’Die hier vereinten Arbeiten eines Jahrzehnts sind Schritte auf meinem Wege zur und in der systematischen Theologie.< Die 18 Beiträge führen in chronologischer Folge von dem die Zeitschrift für Theologie und Kirche unter neuem Herausgeberkreis programmatisch eröffnenden Referat über `Die Bedeutung der historisch-kritischen Methode für die protestantische Theologie und Kircher bis zum Rückblick von Zürich aus auf die denkwürdige Zusammenarbeit mit Hanns Rückert und Ernst Fuchs während der gemeinsamen Tübinger Zeit, denen der Band gewidmet ist. Der Akzent liegt dabei nach beiden Richtungen hin auf der Versicherung, das Grundthema festgehalten zu haben: sowohl angesichts des möglichen Verdachts, als Theologe der Bekennenden Kirche durch das betonte Eintreten für die historisch-kritische Methode eine Abkehr vom bisherigen Weg vollzogen zu haben, wie auch angesichts der räumlichen Trennung von den Freunden das in den gemeinsamen Seminaren über Luthers Paulus-Auslegung hoffnungsvoll Begonnene im Stich zu lassen.

An der kritischen Bezugnahme auf drei namhafte Theologen der Bekennenden Kirche könnte man meinen, für diesen Verdacht Anhalt zu finden. Rudolf Bultmanns Autorität galt der Bekennenden Kirche nicht unbestritten. Aber in seiner entschieden christologisch bestimmten Theologie erblickte man doch eine Befreiung von dem schwankenden Boden der Leben-Jesu-Forschung. Daß ich mich von Bultmann nun gerade in der Frage nach dem historischen Jesus distanzierte, wirkte irritierend, zumal es in der Meinung geschah, auf diese Weise um so besser der christologischen Aufgabe gerecht $\mathrm{zu}$ werden. Diese meine Auffassung gründete auf sorgfältiger Untersuchung des Glaubensbegriffs bei Jesus selbst (Jesus und Glaube, WG I, 203.254), einer sprachlichen Beobachtung, die für mich christologisch wurde. Sie brachte mich auch in Differenz zu K. Barth, der in seiner außerordentlich umfangreichen Lehre von der Versöhnung die explizite Erörterung des Glaubensbegriffs in ungewöhnlich bescheidenem Umfang mit z.T. hämischen Bemerkungen ganz an die Peripherie rückte (s. ebd. 204f., Anm. 2). Und was schließlich das Verhältnis zu Dietrich Bonhoeffer betrifft, so wurde durch das rechte Glaubensverständnis einerseits Sinn geweckt für seine anstössig klingende Rede von der ınichtreligiösen Interpretation biblischer Begriffe`, anderseits vor 
deren missbräuchlicher Verwendung geschützt (s. WG I, 90160).

Als das Grundthema, das trotz und in all dem festgehalten wurde, gab ich an: >die reformatorische Konzentration auf Wort und Glaube als theologische Prinzipienlehre gegenwärtig zu verantworten`. Ich bezog mich dabei auf Luthers Aussage in De captivitate Babylonica ecclesiae sive praeludium, dass Gott mit uns Menschen nie anders gehandelt hat und handelt als durch das Wort derVerheissung und wir wiederum mit Gott nie anders umgehen können als durch den Glauben an sein Verheissungswort (WA 6; 516, 30-32)."

Hier bricht der erste Versuch ab. Es folgen mehrere Seiten handschriftlicher Notizen zu unterschiedlichen Aspekten der Thematik "Wort und Glaube«, gekennzeichnet als "Neuer Versuch". Doch im nächsten Anlauf wird eine zweite Fassung der Einleitung in Angriff genommen, die nun etwas länger wird. In ihr setzt sich Ebeling mit dem Verständnis der Brennpunkte auseinander, bezeichnet sie aber als »Komplexe«:

»Bisher war von den Wegstrecken meiner theologischen Arbeit die Rede, wie sie chronologisch einander folgten. Nun halte ich mich an ein systematisches Ordnungsprinzip, bei dem ich nicht etwa Vollständigkeit anstrebe, sondern mich auf die mehrbändigen Publikationsreihen beschränke. Diese Komplexe bezeichne ich als die Brennpunkte meiner theologischen Arbeit, weil in ihren Titeln das Wesentliche anklingt. Es handelt sich um vier Komplexe. Zum einen die Aufsatzbände ,Wort und Glauber (erschienen 1960, 1969, 1975 und 1995). Sodann die Fundamentaltheologie - als ein Ganzes geplant, aber nur in unfertigen Vorlesungsmanuskripten vorliegend (WS 1972/3, SSD 1973, WS 1973/74, SS 1975 und WS 1978/79). Ferner die Dogmatik des christlichen Glaubens (dreibändig in kurzen Zeitabständen 1979 erschienen). Und schliesslich die Lutherstudien (in fünf Teilbänden: 1971/I; 1977 II,1; 1982 II,2; 1985 [vorgezogen] III; 1989 II,3).

Warum ich die Komplexe in dieser Reihenfolge bespreche, wird sich aus der Darstellung ergeben. Daß ich innerhalb der Publikationsreihen dann doch wieder zeitlich vorgehe, soll daran erinnern, dass die theologische Arbeit nur dann bei der Sache bleibt, wenn sie unterwegs ist."

InVerbindung mit dieser neuen Fassung der Einleitung nimmt Ebeling auch wieder den ersten Brennpunkt in Angriff. Doch diese zwei- 
te Darstellung bricht nach zwei Seiten ab. Sie wird abgelöst von einer dritten, nun etwas ausführlicheren Überarbeitung der Einleitung. Betont wird hier:

"DerVersuch, meine theologische Bemühung nach bestimmten Brennpunkten zu ordnen, geht von der Beobachtung aus, dass meiner theologischen Arbeit selbst eine gewissen Bewegung innewohnt, die es aufzuspüren gilt. Nicht ein Themenkatalog soll zusammengestellt und systematisch gruppiert, vielmehr der Bewegung gefolgt werden, in deren Verlauf sich bestimmte Themen und Fragen anmeldeten. Doch geht es mir nicht um spezielle Gelegenheitsaufgaben, sondern um größere Komplexe, in die sich das Einzelne fügt bzw. aus denen es hervorgeht."

Es ist deutlich erkennbar, dass die Rede von "Brennpunkten" immer problematischer wird. Das gilt vor allem für den vierten, bei dem laut Ebeling schon in der vagen Umschreibung "Orientierung an Luther" eine gewisse Verlegenheit wahrzunehmen sei:

»Im Unterschied zu den erstgenannten >Brennpunkten geht es nun nicht um einen Buchtitel (wie 'Wort und Glaubes) oder eine Disziplin (wie >Fundamentaltheologier oder (Dogmatik (). Man könnte mir vorhalten: Eben dies, eine Theologie Luthers zu schreiben, sei mir nicht gelungen; es habe mir nur zu mancherlei Monographien über ihn gereicht.«

In einem vierten Versuch wird die Schwierigkeit mit dem Begriff "Brennpunkt« noch einmal thematisiert, diesmal grundsätzlicher, als eine Schwierigkeit, die das Vorankommen hemmt:

"Die Schwierigkeit, die mich nicht vorankommen lässt, liegt anscheinend darin, dass die Überschrift `Brennpunkte` in ihrem Bezug unklar ist.

1) bezogen, wie bisher intendiert, auf die Titel der größeren opera: 4 Bde. WuG, mehrbändig geplante Fund.Theol, 3bdg. Dogm, 5 Bd LuSt.

2) Damit aber zugleich die Theol. Hauptthemen intendiert. Das trifft aber nur in formaler Hinsicht zu. Bei den Bd.Reihen WG ist es ein umgreifendes Thema, das so überhaupt nie in diesen Bänden vorkommt. Es gibt keinen Aufsatz über dieses Thema. Man könnte darunter allenfalls eine umfas- 
sende theol. Richtungsangabe verstehen. Aber dann könnte man sie - mit mehr oder weniger Recht, auch auf meine Theologie im ganzen beziehen. Aber trifft das zu?

Die Reflexion mündet in einen Versuch, in den verschiedenen Vorworten der Wort und Glaube-Bände das Entscheidende herauszuarbeiten, was zu mehreren Seiten Notizen führt. Schliesslich schreibt Ebeling an einer fünften Fassung der Einleitung, diesmal interessanterweise unter der Überschrift "B. Schwerpunkte«:

"Indem sich die Darstellung meines theologischen Weges nun einem zweiten Hauptteil zuwendet, folgt sie einem anderen Ordnungsprinzip. Bisher war sie eindeutig biographisch orientiert, und damit am Wechsel von Zeit und Ort. Daraus ergab sich zwangsläufig ein historisches Nacheinander. Unter dem Aspekt des Ortes kann das wohl auch einmal im Hin und Her sein, wie dies in meinem Leben der Fall war. Unter dem Zeitaspekt aber ist nichts zu ändern, nichts rückgängig $\mathrm{zu}$ machen. Bei aller Vorläufigkeit ist es endgültig wie der Lebenslauf. Da lässt sich nichts umdatieren, nichts ungeschehen machen. Das erleichterte die Aufgabe im ersten Teil. Die Reihenfolge stand im grossen ganzen fest. Jetzt geht es eine Darstellung nach bestimmten Sachaspekten. Vereinfachend könnte ich also sagen: nicht biographisch, historisch geordnet, sondern doxographisch, systematisch, nach Fragestellungen, nach Problemen. Ich bezeichne es als vereinfachend, weil auf gängige Ausdrücke zurückgreifend. In Wirklichkeit aber ist es erschwerend, wie eben der Wechsel von historischer zu systematischer Denkweise, von dem nun in Hinsicht auf meinen theologischen Weg die Rede sein soll, und zwar nicht als einen einmaligen biographischen Schritt, sondern als Einschlagen einer anderen theologischen Disziplin. Was ändert sich damit am theologischen Weg? Doch wohl die Strenge, mit der er begangen wird, die Kontrolle, der man sich dabei unterwirft, die Rückhaltlosigkeit, in der man sich mit der Sache der Theologie identifiziert."

Mit diesen grundsätzlichen Überlegungen schliessen die Notizen zum zweiten Teil. Fast scheint es, als koinzidiere der äusserliche Einbruch des Unfalls mit einer internen Schwierigkeit beim Weitermachen: Es will dem Autor anscheinend nicht gelingen, zu einer hermeneutisch überzeugenden Auffassung dessen zu kommen, was ein Brennpunkt 
ist. Die Perspektiven scheinen unklar, uneinheitlich, so dass zunächst von Brennpunkten, dann von Komplexen und schliesslich von Schwerpunkten die Rede ist. Der Versuch weiterzukommen scheitert, und mit diesem Scheitern setzt sich der Autor in immer neuen Anläufen auseinander. Wie Gerhard Ebeling mündlich immer wieder betonte, hätte er diese Schrift gerne noch zu Ende geführt. Insofern hat es etwas Tragisches, wie er hier am Anfang stecken bleibt, nicht weiter kommt. Zugleich ist es aber eindrücklich zu beobachten, wie er sich selbst bis ans Ende treu bleibt: Die Schwierigkeit wird nicht übersprungen, sondern hartnäckig hermeneutisch bearbeitet, auch wenn dies das Vorankommen hemmt. 